\title{
On the convergence to the Nash bargaining solution for endogenous bargaining protocols
}

Citation for published version (APA):

Britz, V., Herings, P. J. J., \& Predtetchinski, A. (2012). On the convergence to the Nash bargaining solution for endogenous bargaining protocols. METEOR, Maastricht University School of Business and Economics. METEOR Research Memorandum No. 030 https://doi.org/10.26481/umamet.2012030

Document status and date:

Published: 01/01/2012

DOI:

10.26481/umamet.2012030

Document Version:

Publisher's PDF, also known as Version of record

\section{Please check the document version of this publication:}

- A submitted manuscript is the version of the article upon submission and before peer-review. There can be important differences between the submitted version and the official published version of record.

People interested in the research are advised to contact the author for the final version of the publication, or visit the DOI to the publisher's website.

- The final author version and the galley proof are versions of the publication after peer review.

- The final published version features the final layout of the paper including the volume, issue and page numbers.

Link to publication

\footnotetext{
General rights rights.

- You may freely distribute the URL identifying the publication in the public portal. please follow below link for the End User Agreement:

www.umlib.nl/taverne-license

Take down policy

If you believe that this document breaches copyright please contact us at:

repository@maastrichtuniversity.nl

providing details and we will investigate your claim.
}

Copyright and moral rights for the publications made accessible in the public portal are retained by the authors and/or other copyright owners and it is a condition of accessing publications that users recognise and abide by the legal requirements associated with these

- Users may download and print one copy of any publication from the public portal for the purpose of private study or research.

- You may not further distribute the material or use it for any profit-making activity or commercial gain

If the publication is distributed under the terms of Article $25 \mathrm{fa}$ of the Dutch Copyright Act, indicated by the "Taverne" license above, 
Volker Britz, P. J ean-J acques Herings, Arkadi Predtetchinski

On the Convergence to the Nash Bargaining Solution for Endogenous Bargaining Protocols

$\mathrm{RM} / 12 / 030$

\section{METEOR}

Maastricht University School of Business and Economics 


\title{
On the Convergence to the Nash Bargaining Solution for Endogenous Bargaining Protocols
}

\author{
Volker Britz $^{1} \quad$ P. Jean-Jacques Herings ${ }^{2} \quad$ Arkadi Predtetchinski ${ }^{3}$
}

June 14, 2012

${ }^{1}$ V. Britz (volker.britz@uclouvain.be), CORE, Université Catholique de Louvain. This author would like to thank the Netherlands Organization for Scientific Research (NWO) for financial support.

${ }^{2}$ P.J.J. Herings (p.herings@maastrichtuniversity.nl), Department of Economics, Maastricht University. This author would like to thank the Netherlands Organization for Scientific Research (NWO) for financial support.

${ }^{3}$ A. Predtetchinski (a.predtetchinski@maastrichtuniversity.nl), Department of Economics, Maastricht University. This author would like to thank the Netherlands Organization for Scientific Research (NWO) for financial support. 


\begin{abstract}
We consider non-cooperative multilateral bargaining games with endogenous bargaining protocols. Under an endogenous protocol, the probability with which a player becomes the proposer in a round of bargaining depends on the identity of the player who previously rejected. An important example is the frequently studied rejector-becomes-proposer protocol. We focus on subgame perfect equilibria in stationary strategies which are shown to exist and to be efficient. Equilibrium proposals do not depend on the probability to propose conditional on the rejection by another player, though equilibrium acceptance sets do depend on these probabilities. Next we consider the limit, as the bargaining friction vanishes. In case no player has a positive probability to propose conditional on his rejection, each player receives his utopia payoff conditional on being recognized and equilibrium payoffs are in general Pareto inefficient. Otherwise, equilibrium proposals of all players converge to a weighted Nash Bargaining Solution, where the weights are determined by the probability to propose conditional on a rejection.
\end{abstract}

Keywords: Strategic Bargaining, Subgame Perfect Equilibrium, Stationary Strategies, Nash Bargaining Solution

JEL CODES: C78 


\section{Introduction}

This paper examines the convergence of equilibrium payoffs in a non-cooperative bargaining game to the asymmetric Nash bargaining solution. In contrast to the existing literature on this topic, we allow for the proposer selection to be endogenous, that is, influenced by the players' actions throughout the game.

We contribute to the so-called Nash program, a research agenda which investigates the relationship between solution concepts from the cooperative (axiomatic) and noncooperative (strategic) branches of game theory. The classic example is the treatment of the bargaining problem by Nash $(1950,1953)$. The bargaining problem here refers to a situation where two players can choose one element of a convex set of feasible payoff pairs by mutual consent. If they fail to agree, an exogenously given pair of disagreement payoffs will realize. The Nash bargaining solution is that payoff pair which maximizes the product of players' gains over their disagreement payoff. Nash (1950) shows that this is the unique bargaining solution satisfying the axioms of scale invariance, symmetry, efficiency, and independence of irrelevant alternatives. Nash (1953) presents a non-cooperative demand game with two players who are uncertain about which payoff pairs are feasible. In the limit as uncertainty vanishes, equilibrium payoffs converge to those predicted by the Nash bargaining solution. In another seminal paper, Rubinstein (1982) also obtains convergence of equilibrium payoffs to the Nash Bargaining Solution, but in a bargaining game with alternating offers. In their discussion of cooperative and non-cooperative approaches to bargaining, Binmore, Rubinstein, and Wolinsky (1986) obtain the Nash bargaining solution in the limit if either players' impatience or the risk of an exogenous breakdown of the negotiations is vanishing.

The asymmetric Nash bargaining solution (Kalai 1977) maximizes a weighted product of players' gains over the disagreement payoffs. It reduces to the Nash bargaining solution when the weights are equal across players. Moreover, it is straightforward to generalize the asymmetric Nash bargaining solution to the case with an arbitrary number of players. However, the analysis of non-cooperative bargaining games with more than two players presents substantial difficulties as long as one insists on unanimity. ${ }^{1}$ In multilateral unanimity bargaining, one typically obtains a wide multiplicity of subgame-perfect equilibrium payoffs. Much of the literature has dealt with this multiplicity by restricting attention to subgame-perfect equilibria in stationary strategies. This restriction allows for a unique

\footnotetext{
${ }^{1}$ There is a branch of literature which circumvents the complications involved in bargaining with more than two players by relaxing the unanimity rule, particularly by allowing partial agreements. Examples of such an approach can be found in Krishna and Serrano (1996), Chae and Yang (1994), and Suh and Wen (2006). In the paper at hand, however, we are interested in situations where a comprehensive and unanimous agreement is required.
} 
prediction of the limit of equilibrium payoff allocations. In this vein, Hart and Mas-Colell (1996) give an early support result for the Nash bargaining solution. Kultti and Vartiainen (2010) consider the extension of the model of Rubinstein (1982) to an arbitrary number of players and assume that proposers rotate according to some fixed order. They show convergence to the Nash bargaining solution in the limit. Extensions of the Rubinstein model where the proposer is selected in each period according to a time-invariant probability distribution, support the asymmetric Nash bargaining solution with the probability distribution as the weight vector as has been demonstrated by Laruelle and Valenciano (2008) and Miyakawa (2008). All these results are special cases of Britz, Herings, and Predtetchinski (2010), who model the proposer selection process as a Markov chain, and obtain convergence to the asymmetric Nash bargaining solution where the weight vector is given by the stationary distribution of the Markov chain. All these proposer selection protocols are exogenous in the sense that the actions of the players in the game have no effect on the identity of the next proposer. To the best of our knowledge, the entire literature that has provided non-cooperative support for the asymmetric Nash bargaining solution considers exogenous protocols only.

Restricting attention to exogenous protocols, however, seems to be a serious limitation. More in particular, the proposer selection may be influenced by the identity of the player who rejects a particular proposal. One simple and intuitively appealing example is the protocol where the player who rejects the current proposal is automatically called to make the next proposal. This rejector-becomes-proposer protocol has been introduced in Selten (1981) and has been studied extensively in both the bargaining and the coalition formation literature, see for example Chatterjee, Dutta, Ray, and Sengupta (1993), Bloch (1996), Ray and Vohra (1999), Imai and Salonen (2000), and Bloch and Diamantoudi (2011) to name a few. Of course, this protocol reduces to Rubinstein's alternating offers model when there are only two players.

The protocol we study in this paper is more general than the rejector-becomes-proposer protocol. Following Kawamori (2008), we are interested in the case where the identity of the player who rejects a proposal may influence the probability by which a particular player becomes the next proposer. Clearly, the rejector-becomes-proposer protocol is a special case of the protocol we consider. Another special case is the protocol where a rejector proposes in the next period with probability zero. It is the polar opposite of the rejectorbecomes-proposer protocol, and can be intuitively justified by the idea that one may want to design a protocol which discourages rejections by punishing them with a zero recognition probability. Moreover, also the protocol with time-invariant recognition probabilities is a special case of the analysis in this paper. In general, however, our protocol does depend on the approval/rejection decisions of the players involved in the bargaining process, and 
is therefore indeed endogenous.

Endogenous protocols are considerably more difficult to analyze than exogenous ones, and the literature has identified a number of cases where both types of protocol lead to surprisingly different results. For instance, Chatterjee, Dutta, Ray, and Sengupta (1993) provide examples for non-existence of equilibria as well as existence of equilibria with delay in the context of an endogenous protocol. On the contrary, it has been shown in Okada (1996) that delay cannot occur at equilibrium and in Okada (2011) that equilibria exist when the protocol is exogenous. Similarly, there are examples for non-existence of equilibrium in Bloch (1996) under an endogenous protocol while Herings and Houba (2010) restore existence for an exogenous proposer selection protocol. Duggan (2011) present a very general coalitional bargaining model where equilibrium existence is shown for exogenous protocols. The paper points out that a similar approach to establish equilibrium existence would not work when the protocol is endogenous.

Our main findings are as follows. We first consider the payoff allocations that are supported by subgame-perfect equilibria in stationary strategies. We show that for any value of the continuation probability such an equilibrium exists, that equilibria are characterized by the absence of delay, and that all equilibrium proposals specify a Pareto efficient payoff allocation, results that would not carry over to the more general environment of coalitional bargaining as evidenced by the examples in Chatterjee, Dutta, Ray, and Sengupta (1993). Equilibrium proposals do not depend on the probability to propose conditional on the rejection by another player, though equilibrium acceptance sets do depend on these probabilities.

Regarding results on the limit of equilibrium payoffs as the continuation probability tends to one, we find a distinction between two cases. If none of the players has a positive probability of being the next proposer after his own rejection, then the proposer in the initial round obtains his utopia payoff, that is his highest payoff in the set of feasible payoffs that satisfy all the individual rationality constraints. Since the initial proposer is selected according to some given probability distribution, the utilities are in general not Pareto efficient, and do therefore not correspond to an asymmetric Nash bargaining solution. Otherwise, we find convergence of the equilibrium payoffs to a weighted Nash bargaining solution, where the weights are determined by the probabilities of making a counter-offer. Players with a zero probability of making a counter-offer will receive a payoff of zero.

The existence of equilibria in stationary strategies and the convergence to a weighted Nash bargaining solution are established results under exogenous protocols and we show that they carry over to our setting with an endogenous protocol if and only if there is at least one player with a strictly positive recognition probability conditional on his rejection. 
However, we see that the bargaining weights depend only on part of the data on the recognition probabilities. In particular, for the limit bargaining equilibrium payoffs it is only relevant with what probability a player proposes after his own rejection. Conversely, it is not important with what probability a player proposes after another player's rejection. Moreover, we find that the payoff allocation in the limit bargaining equilibrium exhibits a discontinuity when the probabilities of making a counter-offer are zero for all players. In that case, the initial proposer has all the bargaining power, and hence the expected payoffs depend on the probability distribution which determines the proposer of the first round.

Romer and Rosenthal (1978) is among the most influential papers emphasizing the role of proposal making on the selected alternative. Kalandrakis (2006) shows in a bargaining framework that the proposer selection process is more important than voting rights, impatience, or complex equilibrium strategies in explaining political power. Empirical support for this feature in the context of the allocation of transportation funds in the US is provided by Knight (2005). The existing results on non-cooperative bargaining games are for exogenous protocols only and do not distinguish between the probability of making a proposal and the probability of making a proposal conditional on a rejection. Our paper argues that the latter probabilities are the ones that really matter to explain bargaining power.

The paper is organized as follows. Section 2 presents the bargaining game with an endogenous protocol. Section 3 presents a characterization of the set of subgame perfect equilibria in stationary strategies. Section 4 introduces the concept of a bargaining equilibrium, a selection of the subgame perfect equilibria in stationary strategies. It is shown that, in payoff terms, the set of bargaining equilibria is equivalent to the set of subgame perfect equilibrium in stationary strategies. It is also shown that a bargaining equilibrium exists. Section 5 studies the case where conditional on his rejection, there is zero probability that a player becomes a proposer, and shows an inefficiency result for this case. Section 6 studies the limit bargaining equilibrium for the complementary case and presents the convergence to the asymmetric Nash bargaining solution. Section 7 presents an example to illustrate the discontinuity in the limit bargaining equilibrium payoffs. Section 8 concludes.

\section{The Bargaining Game}

We consider a bargaining game between finitely many players. The set of players is $N=\{1, \ldots, n\}$. Each player individually can only attain a disagreement payoff which we normalize to zero. However, the players can jointly achieve any payoff vector $v$ in a set $V \subset \mathbb{R}^{n}$ if they unanimously agree on such a payoff vector. Each player is assumed to be an expected utility maximizer. The set $V$ of feasible payoffs and the bargaining protocol 
are the main primitives of the model. We now introduce each in turn.

For vectors $u$ and $v$ in $\mathbb{R}^{n}$, we write $u \geq v$ if $u_{i} \geq v_{i}$ for all $i \in N, u>v$ if $u \geq v$ and $u \neq v$, and $u \gg v$ if $u_{i}>v_{i}$ for all $i \in N$. A point $v$ of $V$ is said to be Pareto-efficient if there is no point $u$ in $V$ such that $u>v$. A point $v$ of $V$ is said to be weakly Pareto-efficient if there is no point $u$ in $V$ such that $u \gg v$. We write $V_{+}$to denote the set $V \cap \mathbb{R}_{+}^{n}$.

Our first assumption is as follows:

[A1] The set $V$ is closed, convex, and comprehensive from below. There is a point $v \in V$ such that $v \gg 0$. The set $V_{+}$is bounded. Each weakly Pareto-efficient point of $V_{+}$is Pareto-efficient.

We will denote by $P$ the set of Pareto-efficient points of $V$ and write $P_{+}$for the set $P \cap \mathbb{R}_{+}^{n}$.

Bargaining takes place in discrete time $t=0,1, \ldots$ There are $n+1$ probability distributions on the players denoted by $\pi^{0}, \pi^{1}, \ldots, \pi^{n}$, each of which belongs to the unit simplex $\Delta^{n}$ in $\mathbb{R}^{n}$.

In round $t=0$, a particular player is chosen as the proposer according to the probability distribution $\pi^{0} \in \Delta^{n}$. The proposer then makes a proposal $v \in V$. Player 1 responds to the proposal by either acceptance or rejection. Once a player $i=1, \ldots, n-1$ has accepted the proposal, it is the turn of player $i+1$ to accept or to reject. ${ }^{2}$ Once player $n$ has accepted the proposal, the game ends and the approved proposal is implemented.

As soon as some player $j \in N$ rejects a proposal in round $t$, the game ends with probability $1-\delta>0$ and payoffs to all players are zero. With the complementary probability $\delta$, the game continues to round $t+1$. The proposer in that round is then drawn from the probability distribution $\pi^{j}$. If the game continues perpetually without agreement, the payoff to every player is zero.

In case $\pi^{0}, \pi^{1}, \ldots, \pi^{n}$ all coincide, we are back in the familiar case of an exogenous protocol with time-invariant recognition probabilities. The rejector-becomes-proposer protocol follows from specifying $\pi_{i}^{i}=1$ for all $i \in N$. A polar opposite of the rejector-becomesproposer protocol, where a rejector proposes with probability zero in the next round, follows by setting $\pi_{i}^{i}=0$ for all $i \in N$.

It is well-known that bargaining games with more than two players admit a wide multiplicity of subgame perfect equilibria (SPE), see Herrero (1985) and Haller (1986). We will therefore restrict attention to SPE in stationary strategies (SSPE). A stationary strategy for player $i$ consists of a proposal $\theta^{i} \in V$ which $i$ makes whenever it is his turn to propose

\footnotetext{
${ }^{2}$ Throughout the paper, for the sake of simplicity, we assume that players respond to a proposal in the fixed order $1, \ldots, n$. We show in the appendix that all results carry over to the case with arbitrary voting orders.
} 
and an acceptance set $A^{i} \subset V$ which consists of all the proposals which player $i$ would be willing to accept if they were offered to him. We denote the social acceptance set by $A=\cap_{i \in N} A^{i}$ and write the profile of stationary strategies $\left(\theta^{1}, A^{1}, \ldots, \theta^{n}, A^{n}\right)$ more concisely as $(\Theta, \mathcal{A})$.

\section{$3 \quad$ Subgame Perfect Equilibria in Stationary Strategies}

In this section, we consider the set of SSPEs of the bargaining game. Fix some profile of stationary strategies $(\Theta, \mathcal{A})$. By definition of a stationary strategy, there is a unique payoff vector which is expected in any subgame following a rejection by some player $i \in N$. We refer to it as the vector of continuation payoffs after $i$ 's rejection and denote it by $q^{i}(\Theta, \mathcal{A})$. Since it will be clear from the context, we omit the argument in the sequel. Moreover, we define a vector $r(\Theta, \mathcal{A})$ of reservation payoffs by $r(\Theta, \mathcal{A})=\left(q_{1}^{1}, \ldots, q_{n}^{n}\right)$. Again, we will omit the argument in the sequel. It is important to note at this stage that the endogenous protocol which we consider induces a disparity between the continuation and the reservation payoffs. Depending on the identity of the player who rejects the current proposal, one out of $n$ different continuation payoff vectors will realize. When these $n$ vectors are thought of as the columns of a matrix, the reservation payoffs correspond to its diagonal. If the protocol is exogenous, all the columns of this matrix are identical since the sequel of the game is then not influenced by the identity of the rejector. We will see that the reservation payoffs are important as an "acceptance threshold" in a sense to be made precise in the next two lemmas.

For every $i \in N$, let $S(i)=\{j \in N \mid j \geq i\}$. That is, $S(i)$ is the set of players succeeding player $i$ in the response order, including $i$ himself. We denote $\cap_{j \in S(i)} A^{j}$ by $A^{S(i)}$.

Lemma 3.1 Let $(\Theta, \mathcal{A})$ be an SSPE inducing reservation payoffs $r$. It holds that

1. If $v \in V$ is such that $v_{n}>r_{n}$, then $v \in A^{n}$.

2. For every $i=1, \ldots, n-1$, if $v \in A^{S(i+1)}$ and $v_{i}>r_{i}$, then $v \in A^{i}$.

Proof: Consider a history of the game where player $n$ has to respond to the proposal $v$ with $v_{n}>r_{n}$. If player $n$ accepts, the proposal passes and he receives $v_{n}$. If he rejects, he expects to receive $r_{n}$, so he would have a profitable deviation at this history. This establishes the first part of the lemma. Now consider a history of the game where player $i \in N \backslash\{n\}$ has to respond to the proposal $v$ with $v_{i}>r_{i}$ and $i \in A^{S(i+1)}$. If player $i$ accepts $v$, this proposal passes and he receives $v_{i}$. Otherwise, he receives $r_{i}$, so he would have a profitable deviation at this history. 
Lemma 3.1 implies that a proposal lies in the social acceptance set if all players receive strictly higher utility from this proposal than from the vector $r$ of reservation payoffs.

Corollary 3.2 Let $(\Theta, \mathcal{A})$ be an SSPE inducing reservation payoffs $r$. If $v \gg r$, then $v \in A$.

We consider next a necessary condition for proposals to be accepted.

Lemma 3.3 Let $(\Theta, \mathcal{A})$ be an SSPE inducing reservation payoffs $r$. For every $i=1, \ldots, n$, if $v \in A^{S(i)}$, then $v_{j} \geq r_{j}$ for all $j \in S(i)$.

Proof: Suppose by way of contradiction that $v \in A^{S(i)}$ but there is $j \geq i$ such that $v_{j}<r_{j}$. If player $j$ unilaterally deviates to reject rather than to accept the proposal $v$, he receives a payoff of $r_{j}$. This deviation is profitable.

Lemma 3.3 is a slightly weaker statement than the converse of Lemma 3.3. It says that if a proposal gives at least one player strictly less than his reservation payoff, then that proposal will not be unanimously accepted.

Corollary 3.4 Let $(\Theta, \mathcal{A})$ be an SSPE inducing reservation payoffs $r$. For all $v \in A$ it holds that $v \geq r$.

The two foregoing statements leave some indeterminacy with regard to the accept/reject decisions of individual players; we address this issue in detail later on. With the above lemmas, we have shown how exactly the vector $r$ plays the role of an acceptance threshold. The next lemma derives the implication that an agreement can be reached only if $r$ belongs to the set $V_{+}$.

Lemma 3.5 Let $(\Theta, \mathcal{A})$ be an SSPE inducing reservation payoffs $r$. Then there exists a $v \in V$ such that $v \gg r \geq 0$. In particular, it holds that $A \neq \emptyset$ and $r \in V_{+}$.

Proof: Any player $i$ can choose to reject all proposals, a strategy that never leads to an agreement irrespective of the strategy used by the other players, and a payoff of zero for all players. It follows that player $i$ 's payoff in any subgame perfect equilibrium cannot be smaller than zero. In particular, it follows that $r \geq 0$.

Suppose now that there is no $v \in V$ such that $v \gg r$. In view of Assumption A1, there is no $v \in V$ such that $v>r$. It now follows from Corollary 3.4 that $A \subset\{r\}$. First suppose that $A=\emptyset$. In this case equilibrium strategies lead to payoffs of zero for all players, so $r=0$. But under Assumption A1 there is a vector $v \in V$ with $v \gg 0$, a contradiction to our supposition. Hence $A=\{r\}$. 
Then, after a rejection, only two outcomes are possible: Either agreement on $r$ is reached at some future time or zero payoffs result. The vector of players' continuation payoffs after a rejection is therefore a convex combination of 0 and $r$, where the former has a weight of at least $1-\delta$. But this implies $r_{i} \leq \delta r_{i}$ for all $i \in N$. Since $\delta<1$, we conclude that $r=0$. As before, this leads to a contradiction.

Lemma 3.5 implies that at an SSPE a proposer is always able to make a proposal which gives all players a strictly higher payoff than their reservation payoffs, and which will therefore be accepted. However, with the endogenous protocol under consideration here, it is not immediate that the proposer finds it in his best interest to make such an acceptable proposal. This complication is due to the disparity which exists in our model between the continuation payoffs $q^{1}, \ldots, q^{n}$ and the reservation payoffs $r$. In particular, if some continuation payoff $q_{i}^{j}$ is high enough, one might conjecture that player $i$ would prefer making a proposal which will be rejected by player $j$ rather than make an acceptable proposal. The next step in our argument is to show that such behavior is not consistent with SSPE. More precisely, we will show in the next lemma that each player's SSPE proposal is acceptable and therefore SSPEs lead to immediate agreement.

Lemma 3.6 Let $(\Theta, \mathcal{A})$ be an SSPE. For all $i \in N$ it holds that $\theta^{i} \in A$ and $\theta_{i}^{i}>0$.

Proof: Let $u_{i}$ be the SSPE utility to player $i$ conditional on reaching a history where it is player $i$ 's turn to make a proposal. It holds that $u_{i}=\theta_{i}^{i}$ if $\theta^{i} \in A$ and $u_{i}=q_{i}^{j}$ if $\theta^{i} \notin A$, where $j$ is the least element of $N$ such that $\theta^{i} \notin A^{j}$.

By making a proposal $v \in A$, player $i$ guarantees himself a payoff of $v_{i}$. It follows that $u_{i} \geq v_{i}$ for every $v \in A$. In particular $u_{i}>0$ since Corollary 3.2 and Lemma 3.5 imply that there is a vector $v \in A$ such that $v_{i}>0$.

Let $U$ be the set of proposals $\theta^{k}$ for $k \in N$ that belong to the social acceptance set $A$, along with the vector 0 . Following the rejection of player $i$ 's proposal $\theta^{i}$ by player $j$, the vector $q^{j}$ of continuation payoffs is a convex combination of the vectors in $U$, with 0 having a weight of at least $1-\delta$. We know from the preceding paragraph that $u_{i} \geq \theta_{i}^{k}$ for all $\theta^{k} \in U$ and that $u_{i}>0$. It follows that $u_{i}>q_{i}^{j}$. But this implies that player $i$ 's equilibrium proposal $\theta^{i}$ is not rejected by player $j$, i.e. $\theta^{i} \in A$, establishing the first claim. Moreover, $\theta_{i}^{i}=u_{i}>0$.

The previous lemma implies that immediate agreement will be reached in an SSPE. In the following lemma, we show that the proposer always gives other players their reservation payoffs and then maximizes his own payoff.

Lemma 3.7 Let $(\Theta, \mathcal{A})$ be an SSPE inducing reservation payoffs $r$. For every $i \in N$, it holds that $\theta^{i} \in P_{+}$and $\theta_{j}^{i}=r_{j}$ for all $j \in N \backslash\{i\}$. 
Proof: We show first that $\theta^{i} \in P_{+}$for all $i \in N$. By Corollary 3.4, Lemma 3.5, and Lemma 3.6 we have $\theta^{i} \geq r \geq 0$. In view of Assumption A1 it suffices to prove that $\theta^{i}$ is weakly Pareto-efficient. Suppose to the contrary. Then there is a vector $v \in V$ such that $v \gg \theta^{i}$. Hence $v \gg r$ and so by Corollary $3.2 v \in A$. At a history where player $i$ is the proposer, he has a profitable deviation by proposing $v$ instead of $\theta^{i}$ since $v_{i}>\theta_{i}^{i}$. The contradiction establishes the first part of the claim.

We prove that each responder receives exactly his reservation payoff. Define the vector $v$ by $v_{i}=\theta_{i}^{i}$ and $v_{j}=r_{j}$ for every $j \in N \backslash\{i\}$. Then $\theta^{i} \geq v \geq r$. Suppose, contrary to our claim, that there is a player $j \in N \backslash\{i\}$ such that $\theta_{j}^{i}>r_{j}$. Then $\theta_{j}^{i}>v_{j}$, and hence $v$ is not Pareto-efficient. In view of our Assumption A1, $v$ is not weakly Pareto-efficient. So there exists a vector $v^{\prime} \in V$ with $v^{\prime} \gg v$. But then $v^{\prime} \gg r$ so by Corollary 3.2 it holds that $v^{\prime} \in A$. Since in particular $v_{i}^{\prime}>v_{i}=\theta_{i}^{i}$, proposing the vector $v^{\prime}$ instead of $\theta^{i}$ is a profitable deviation for player $i$.

For every $i \in N$, we define

$$
\alpha_{i}=\frac{\delta \pi_{i}^{i}}{1-\delta+\delta \pi_{i}^{i}}
$$

Corollary 3.8 Let $(\Theta, \mathcal{A})$ be an SSPE inducing reservation payoffs $r$. For all $i \in N$ it holds that $r_{i}=\alpha_{i} \theta_{i}^{i}$.

Proof: Since each proposal belongs to the social acceptance set, the reservation payoffs can be computed as follows:

$$
r_{i}=\delta \sum_{j=1}^{n} \pi_{j}^{i} \theta_{i}^{j}=\delta \pi_{i}^{i} \theta_{i}^{i}+\delta\left(1-\pi_{i}^{i}\right) r_{i} .
$$

Solving for $r_{i}$ yields the desired expression.

In the following theorem, we collect all the necessary conditions that we have derived so far for a strategy profile to be an SSPE.

Theorem 3.9 If $(\Theta, \mathcal{A})$ is an SSPE inducing reservation payoffs $r$, then

$$
\begin{aligned}
& A^{S(i)} \subset \cap_{j=i, \ldots, n}\left\{v \in V \mid v_{j} \geq r_{j}\right\}, \quad i \in N, \\
& A^{n} \supset\left\{v \in V \mid v_{n}>r_{n}\right\}, \\
& A^{i} \supset\left\{v \in A^{S(i+1)} \mid v_{i}>r_{i}\right\}, \quad i \in N \backslash\{n\}, \\
& \theta_{j}^{i}=r_{j}, \quad i \in N, j \in N \backslash\{i\}, \\
& r_{i}=\alpha_{i} \theta_{i}^{i}, \quad i \in N, \\
& \theta^{i} \in P_{+} \cap A, \quad i \in N .
\end{aligned}
$$


One important implication of the foregoing analysis is that a responding player who does not have positive probability of making a counter-offer will not be offered a positive payoff. Indeed, if $\pi_{i}^{i}=0$ for some player $i$, then $\alpha_{i}=0$ and so $r_{i}=0$.

Corollary 3.10 Let $(\Theta, \mathcal{A})$ be an SSPE inducing reservation payoffs $r$ and let $i \in N$ be a player with $\pi_{i}^{i}=0$. Then it holds that $r_{i}=0$ and $\theta_{j}^{i}=0$ for all $j \in N \backslash\{i\}$.

We define the utopia payoff $\hat{v}_{i}$ of a player $i \in N$ as the highest payoff in $V$ player $i$ can obtain subject to the individual rationality constraints, that is $\hat{v}_{i}=\max \left\{v_{i} \mid v \in V_{+}\right\}$. Let $\hat{v}=\left(\hat{v}_{1}, \ldots, \hat{v}_{n}\right)$. Consider the case where all players have zero probability to make a counter-offer conditional on a rejection. That is, suppose $\pi_{i}^{i}=0$ for all $i \in N$. In that case, Theorem 3.9 readily implies that at any history where player $i$ is the proposer, he receives his utopia payoff in an SSPE.

Corollary 3.11 Assume $\pi_{i}^{i}=0$ for all $i \in N$. In any SSPE, the payoff to player $i \in N$ is equal to $\pi_{i}^{0} \hat{v}_{i}$.

\section{Bargaining Equilibrium}

In this section we show that SSPEs exist. To do so, we will show the existence of a particular type of SSPE called bargaining equilibrium. We will also argue that in terms of payoffs nothing is lost by restricting attention to bargaining equilibria.

Consider proposals $\Theta \in V_{+}^{n}$. We define acceptance sets $\mathcal{A}(\Theta)$ as a function of these proposals. We begin with the acceptance set for player $n$ and then proceed inductively to define the acceptance sets of players $n-1, \ldots, 1$. Indeed, let

$$
A^{n}(\Theta)=\left\{v \in V \mid v_{n} \geq \alpha_{n} \theta_{n}^{n}\right\} .
$$

Now suppose that for some player $i \in N \backslash\{n\}$, the acceptance set $A^{j}(\Theta)$ has been defined for all $j \in S(i+1)$. For any $v \in V$, we define player $i$ 's payoff upon acceptance of $v$ as follows:

$$
\beta_{i}(v)= \begin{cases}v_{i}, & \text { if } v \in \bigcap_{j=i+1}^{n} A^{j}(\Theta), \\ \delta \theta_{i}^{i}\left(\frac{(1-\delta) \pi_{i}^{k}+\delta \pi_{i}^{i}}{1-\delta+\delta \pi_{i}^{i}}\right), & \text { if } v \in \bigcap_{j=i+1}^{k-1} A^{j}(\Theta) \backslash A^{k}(\Theta) \text { for some } k \in S(i+1),\end{cases}
$$

where $\cap_{j=i+1}^{k-1} A^{j}(\Theta)$ is equal to $\mathbb{R}^{n}$ by definition if $k=i+1$. As will become clear from the subsequent discussion, the payoff $\beta_{i}(v)$ corresponds to the payoff to player $i$ from accepting the proposal $v$. We define

$$
A^{i}(\Theta)=\left\{v \in V \mid \beta_{i}(v) \geq \alpha_{i} \theta_{i}^{i}\right\} .
$$


The construction of acceptance sets $\mathcal{A}(\Theta)$ is now complete. We denote by $A(\Theta)$ the concomitant social acceptance set, that is, the intersection of the sets $A^{i}(\Theta), i=1, \ldots, n$.

Definition 4.1 A bargaining equilibrium is a strategy profile $(\Theta, \mathcal{A}(\Theta))$ such that for all $i \in N$,

$$
\begin{aligned}
& \theta^{i} \in P_{+}, \\
& \theta_{j}^{i}=\alpha_{j} \theta_{j}^{j}, \quad j \in N \backslash\{i\} .
\end{aligned}
$$

Since the coefficients $\alpha_{i}$ do not depend on $\pi_{j}^{i}$ for $j \neq i$, we see that the equilibrium proposals in a bargaining equilibrium do not depend on the probability to propose conditional on the rejection by another player. As is clear from the construction above, equilibrium acceptance sets do depend on these probabilities.

We show next that in a bargaining equilibrium there is no delay before reaching an agreement.

Theorem 4.2 In a bargaining equilibrium $(\Theta, \mathcal{A}(\Theta))$, agreement is reached immediately: for all $i \in N, \theta^{i} \in A(\Theta)$.

Proof: We prove the result by induction. For $i \in N \backslash\{n\}$, we have $\theta_{n}^{i}=\alpha_{n} \theta_{n}^{n}$, so $\theta^{i} \in A^{n}(\Theta)$. For $i=n$, since $\alpha_{n}<1$, we have $\theta_{n}^{n} \geq \alpha_{n} \theta_{n}^{n}$, so $\theta^{n} \in A^{n}(\Theta)$.

Now suppose that for some player $j \in N \backslash\{n\}$ it holds that $\theta^{i} \in A^{k}(\Theta)$ for all $k \in$ $S(j+1)$. We show that $\theta^{i} \in A^{j}(\Theta)$ thereby completing the proof.

By the induction hypothesis it holds that $\beta_{j}\left(\theta^{i}\right)=\theta_{j}^{i}$. For $i \in N \backslash\{j\}$, we have

$$
\beta_{j}\left(\theta^{i}\right)=\theta_{j}^{i}=\alpha_{j} \theta_{j}^{j},
$$

so $\theta^{i} \in A^{j}(\Theta)$. For $i=j$, since $\alpha_{j}<1$, we have

$$
\beta_{j}\left(\theta^{j}\right)=\theta_{j}^{j} \geq \alpha_{j} \theta_{j}^{j}
$$

so $\theta^{j} \in A^{j}(\Theta)$.

Since bargaining equilibrium proposals do not depend on the probability to propose conditional on the rejection by another player, and equilibrium proposals are accepted without delay, it follows that the equilibrium utility depends on the initial recognition probabilities $\pi^{0}$ and on the probability to propose conditional on an own rejection $\pi_{i}^{i}$, but not on the probabilities $\pi_{j}^{i}$ for $j \neq i$.

Another important feature of a bargaining equilibrium is that there is an advantage to being the proposer. To see this, simply notice that $\alpha_{i}<1$, so that $\theta_{j}^{i}<\theta_{i}^{i}$ for $j \neq i$. 
The continuation payoff to player $i$ after a rejection of a proposal by player $k$ can be computed as follows:

$$
\begin{aligned}
q_{i}^{k}(\Theta, \mathcal{A}(\Theta)) & =\delta \sum_{j=1}^{n} \pi_{j}^{k} \theta_{i}^{j}=\delta \pi_{i}^{k} \theta_{i}^{i}+\delta\left(1-\pi_{i}^{k}\right)\left(\frac{\delta \pi_{i}^{i}}{1-\delta+\delta \pi_{i}^{i}}\right) \theta_{i}^{i} \\
& =\delta \theta_{i}^{i}\left(\frac{(1-\delta) \pi_{i}^{k}+\delta \pi_{i}^{i}}{1-\delta+\delta \pi_{i}^{i}}\right) .
\end{aligned}
$$

The reservation payoffs are given by

$$
r_{i}(\Theta, \mathcal{A}(\Theta))=q_{i}^{i}(\Theta, \mathcal{A}(\Theta))=\alpha_{i} \theta_{i}^{i} .
$$

In a bargaining equilibrium, each responder receives precisely his reservation payoff. The payoff $\beta_{i}(v)$ upon acceptance of $v$ is $v_{i}$ if $v \in A^{j}(\Theta)$ for every player $j \in S(i+1)$ and it is $q_{i}^{k}$ otherwise, where $k$ is the lowest indexed player in $S(i+1)$ with $v \notin A^{k}(\Theta)$. Thus $\beta_{i}(v)$ is the payoff to $i$ from accepting the proposal $v$. Player $i$ accepts the proposal $v$ if and only if $\beta_{i}(v) \geq r_{i}$.

For $v$ to be an element of the social acceptance set $A(\Theta)$ in a bargaining equilibrium, it should hold that $v_{i} \geq \alpha_{i} \theta_{i}^{i}$ for all $i \in N$. Indeed, when $v \in A(\Theta)$, we have that $\beta_{i}(v)=v_{i}$ for all $i \in N$, so $v \in A^{i}(\Theta)$ implies $v_{i} \geq \alpha_{i} \theta_{i}^{i}$ for all $i \in N$. It is also easily verified that the converse is true, if $v_{i} \geq \alpha_{i} \theta_{i}^{i}$ for all $i \in N$, then $v \in A(\Theta)$. It follows that in a bargaining equilibrium $(\Theta, \mathcal{A}(\Theta))$,

$$
A(\Theta)=\left\{v \in V \mid \forall i \in N, v_{i} \geq \alpha_{i} \theta_{i}^{i}\right\} .
$$

We now discuss the relationship between SSPE and bargaining equilibrium. We show that each bargaining equilibrium is an SSPE. While an SSPE need not be a bargaining equilibrium, it induces one in a natural way. Intuitively, a bargaining equilibrium can be thought of as an SSPE that breaks the indifference in the accept/reject decisions in favor of acceptance. Our results imply that in as much as we are interested in equilibrium proposals and payoffs rather than acceptance sets, there is no loss in focussing the analysis on the set of bargaining equilibria rather than the set of SSPEs.

Theorem 4.3 A bargaining equilibrium is an SSPE.

Proof: Let $(\Theta, \mathcal{A}(\Theta))$ be a bargaining equilibrium. By definition, the bargaining equilibrium is a profile of stationary strategies. We show that it is a subgame perfect equilibrium. Applying the one-shot deviation property, we have to show that at any history the acting player has no profitable one-shot deviation.

Consider first a one-shot deviation in the accept/reject decisions. By construction of $\mathcal{A}(\Theta)$, a responder accepts a proposal if and only if accepting makes him weakly better off than rejecting. Hence, a one-shot deviation cannot be profitable. 
Now consider a one-shot deviation by player $i$, who proposes some $v \in V \backslash\left\{\theta^{i}\right\}$. Suppose that $v \notin A(\Theta)$. Then, the expected payoff to player $i$ from proposing $v$ is $q_{i}^{k}$ where $k$ is the first player in the response order to reject $v$. It follows from the expression for the continuation payoffs given above that

$$
q_{i}^{k}=\delta \theta_{i}^{i}\left(\frac{(1-\delta) \pi_{i}^{k}+\delta \pi_{i}^{i}}{1-\delta+\delta \pi_{i}^{i}}\right) \leq \delta \theta_{i}^{i} \leq \theta_{i}^{i}
$$

so the deviation is not profitable. Now consider the case where $v \in A(\Theta)$. Then $v_{j} \geq \alpha_{j} \theta_{j}^{j}$ for every $j \in N$ and hence $v_{j} \geq \theta_{j}^{i}$ for every $j \in N \backslash\{i\}$. Since the vector $\theta^{i}$ is Paretoefficient, we must have $v_{i} \leq \theta_{i}^{i}$. Again, the deviation is not profitable.

Theorem 4.4 If $(\Theta, \mathcal{A})$ is an SSPE, then $(\Theta, \mathcal{A}(\Theta))$ is a bargaining equilibrium.

Proof: Condition (4.1) of Definition 4.1 is implied by Condition (3.6) of Theorem 3.9. Condition (4.2) is implied by Conditions (3.4)-(3.6) of Theorem 3.9.

By Condition (3.6) of Theorem 3.9 it holds that there is immediate agreement in any SSPE. It therefore follows from Theorems 4.3 and 4.4 that the distinction between SSPE and bargaining equilibrium is immaterial. The set of SSPE utilities coincides with the set of bargaining equilibrium utilities. It follows in particular that SSPE utilities depend on the initial recognition probabilities $\pi^{0}$ and on the probability to propose conditional on an own rejection $\pi_{i}^{i}$, but not on the probabilities $\pi_{j}^{i}$ for $j \neq i$.

Theorem 4.5 A bargaining equilibrium exists.

Proof: Let us define $\rho \in \Delta^{n}$ and $\lambda \in[0,1)$ as follows. If $\pi_{i}^{i}>0$ for at least one $i \in N$, then

$$
\begin{aligned}
\rho_{i} & =\frac{\pi_{i}^{i}}{\sum_{j=1}^{n} \pi_{j}^{j}}, \quad i \in N, \\
\lambda & =\frac{\delta \sum_{i=1}^{n} \pi_{i}^{i}}{1-\delta+\delta \sum_{i=1}^{n} \pi_{i}^{i}} .
\end{aligned}
$$

If $\pi_{i}^{i}=0$ for all $i \in N$, then we set $\lambda=0$ and $\rho_{i}=\frac{1}{n}$ for all $i \in N$. After elementary calculus, it follows from Definition 4.1 that the system of characteristic equations describing bargaining equilibrium proposals of our model with recognition probabilities $\pi^{0}, \pi^{1}, \ldots, \pi^{n}$ and continuation probability $\delta$ coincides with the system of characteristic equations which describes equilibrium proposals in a bargaining model with time-invariant recognition probabilities $\rho$ and continuation probability $\lambda$. The existence of a solution to the latter system has been shown by Banks and Duggan (2000) in their Theorems 1 and $2 .^{3}$

\footnotetext{
${ }^{3}$ In Banks and Duggan (2000) the continuation probability is 1, and players have time preferences with a discount factor equal to $\delta$. Voting is simultaneous rather than sequential, and attention is restricted to stage-undominated voting strategies. For the case with time-invariant recognition probabilities, both modeling choices lead to exactly the same equilibrium conditions on proposals as in Definition 4.1.
} 
We have shown that a bargaining equilibrium is an SSPE, so the existence of an SSPE is implied by the theorem above.

Corollary 4.6 An SSPE exists.

\section{No Possibility to Propose after a Rejection}

In this section we study the case where conditional on a rejection there is zero probability to be selected as a proposer, so $\pi_{i}^{i}=0$ for every player $i \in N$. Corollary 3.11 states that in any SSPE the payoff to player $i \in N$ is equal to $\pi_{i}^{0} \hat{v}_{i}$. Notice that this expression is independent of the continuation probability $\delta$. Moreover, in case $P_{+}$is not a simplex, i.e. $P_{+}$cannot be written as the convex hull of $n$ extreme points, and each player has a positive probability to be the initial proposer, equilibrium payoffs are in the interior of the set $V$ and therefore inefficient.

Theorem 5.1 Assume $P_{+}$is not a simplex, $\pi_{i}^{i}=0$ for all $i \in N$, and $\pi^{0} \gg 0$. All SSPEs are inefficient.

Proof: By Corollary 3.11 it holds that SSPE utilities are equal to $\sum_{i \in N} \pi_{i}^{0} \hat{v}_{i} e(i)$, where $e(i)$ denotes the $i$-th unit vector in $\mathbb{R}^{n}$. Since $P_{+}$is not a simplex, there is $p \in$ $P_{+} \backslash \operatorname{conv}\left(\left\{\hat{v}_{i} e(i) \mid i \in N\right\}\right)$, where $\operatorname{conv}(X)$ denotes the convex hull of a set of points $X$. Choose weights $\lambda_{i} \in \mathbb{R}, i \in N$, such that $p=\sum_{i \in N} \lambda_{i} \hat{v}_{i} e(i)$. Since $p \geq 0$, it holds that $\lambda_{i} \geq 0$ for all $i \in N$. It then follows that $\sum_{i \in N} \lambda_{i}>1$, since $\sum_{i \in N} \lambda_{i}<1$ contradicts the Pareto optimality of $p$, and $\sum_{i \in N} \lambda_{i}=1$ implies $p \in \operatorname{conv}\left(\left\{\hat{v}_{i} e(i) \mid i \in N\right\}\right)$, leading to a contradiction as well.

Take $\gamma>0$ sufficiently small such that $\pi^{0}-\gamma \lambda \geq 0$. Notice that non-negativity of $\pi^{0}-\gamma \lambda$ implies that $\gamma<1$. We define $\mu \in \Delta$ by

$$
\mu=\frac{1}{1-\gamma}\left(\pi^{0}-\gamma \lambda\right)+\frac{\gamma\left(\sum_{i \in N} \lambda_{i}-1\right)}{1-\gamma} e(1),
$$

and $v \in \operatorname{conv}\left(\left\{\hat{v}_{i} e(i) \mid i \in N\right\}\right) \subset V$ by

$$
v=\sum_{i \in N} \mu_{i} \hat{v}_{i} e(i)
$$

By convexity of $V$ it holds that $\gamma p+(1-\gamma) v \in V$. Straightforward calculus shows that

$$
\gamma p+(1-\gamma) v=\left(\pi_{1}^{0}+\gamma\left(\sum_{i \in N} \lambda_{i}-1\right)\right) \hat{v}_{1} e(1)+\sum_{i \in N \backslash\{1\}} \pi_{i}^{0} \hat{v}_{i} e(i) .
$$

Since $\sum_{i \in N} \lambda_{i}-1>0$ and $\gamma>0$ it holds that $\gamma p+(1-\gamma) v$ is a point in $V$ which Pareto dominates the vector of SSPE utilities. 
Although at first glance it might appear as a good idea to deny players the right to make a proposal following a rejection, Theorem 5.1 claims that the contrary is the case. Since such protocols give all bargaining power to the initial proposer, the equilibrium result is a lottery where player $i$ receives his utopia payoff $\hat{v}_{i}$ with probability $\pi_{i}^{0}$ and a payoff of 0 with probability $1-\pi_{i}^{0}$, leading to inefficient equilibrium utilities when players are risk-averse, or, more generally, when $P_{+}$is not a simplex.

\section{The Limit Bargaining Equilibrium}

In this section, we study the case where at least one player has a positive probability to make a counter-offer conditional on a rejection, so there is a player $i \in N$ with $\pi_{i}^{i}>0$. Moreover, we focus on the convergence of bargaining equilibrium payoffs as the bargaining friction vanishes, i.e. as the continuation probability $\delta$ tends to one. We employ the additional assumption A2, which is imposed throughout this section without further mentioning.

A vector $\eta$ in $\mathbb{R}^{n}$ is a normal vector to $V$ at a point $v \in V$ if $(u-v)^{\top} \eta \leq 0$ for all $u \in V$. It is said to be a unit normal vector if $\|\eta\|=1$.

[A2] There is a continuous function $\eta: P_{+} \rightarrow \mathbb{R}^{n}$ such that $\eta(v)$ is a unit normal vector to $V$ at the point $v$.

Assumption A2 implies that the boundary $P_{+}$does not have kinks. Notice that in view of Assumption A1 we have $\eta_{i}(v)>0$ for every $i \in N$ such that $v_{i}>0$. All the existing results on the convergence of multi-lateral bargaining to the weighted Nash bargaining solution for exogenous protocols make Assumption A2. Indeed, without such an assumption, Kultti and Vartiainen (2010) provide an example showing that the unique bargaining equilibrium may not converge to the Nash bargaining solution and Herings and Predtetchinski (2011) show that the limit of bargaining equilibria may not be unique.

Lemma 6.1 Let $\left(\delta_{m}\right)_{m \in \mathbb{N}}$ be a sequence of continuation probabilities converging to 1 from below. For every $m \in \mathbb{N}$, let $\left(\theta_{m}^{1}, \ldots, \theta_{m}^{n}\right)$ be bargaining equilibrium proposals of the game with continuation probability $\delta_{m}$. If the sequence $\left(\theta_{m}^{1}, \ldots, \theta_{m}^{n}\right)$ converges to $\left(\bar{\theta}^{1}, \ldots, \bar{\theta}^{n}\right)$, then $\bar{\theta}^{1}=\cdots=\bar{\theta}^{n}$. Moreover, for every $i \in N$, the point $\bar{\theta}^{i}$ is Pareto-efficient.

Proof: We prove first that for every $i \in N$ the point $\bar{\theta}^{i}$ is Pareto-efficient. Suppose not. Then, in view of Assumption A1, there is $i \in N$ and $v \in V$ such that $v \gg \bar{\theta}^{i}$. But then $v \gg \theta_{m}^{i}$ for $m$ large enough, which contradicts the definition of bargaining equilibrium where it is required that $\theta_{m}^{i} \in P_{+}$.

Take $i \in N$ such that $\pi_{i}^{i}>0$ and take any other player $j \in N$. We wish to show that $\bar{\theta}^{j}=\bar{\theta}^{i}$. For every $m \in \mathbb{N}$, for every $k \in N \backslash\{i, j\}$ it holds that $\theta_{m, k}^{i}=\theta_{m, k}^{j}=\alpha_{m, k} \theta_{m, k}^{k}$. 
Taking the limit as $m$ goes to infinity yields $\bar{\theta}_{k}^{i}=\bar{\theta}_{k}^{j}$. Furthermore, for every $m \in \mathbb{N}$, we have $\theta_{m, i}^{j}=\alpha_{m, i} \theta_{m, i}^{i}$. Since $\alpha_{m, i}$ converges to one as $m$ tends to infinity, we obtain $\bar{\theta}_{i}^{j}=\bar{\theta}_{i}^{i}$. We conclude that the vectors $\bar{\theta}^{i}$ and $\bar{\theta}^{j}$ can only differ in component $j$. Since both points $\bar{\theta}^{i}$ and $\bar{\theta}^{j}$ are Pareto-efficient, we have $\bar{\theta}^{i}=\bar{\theta}^{j}$, as desired.

The lemma above therefore justifies the following definition.

Definition 6.2 A limit bargaining equilibrium is a proposal $\theta \in V$ for which there exist sequences $\left(\delta_{m}\right)_{m \in \mathbb{N}}$ and $\left(\theta_{m}^{1}, \ldots, \theta_{m}^{n}\right)_{m \in \mathbb{N}}$, where $\left(\theta_{m}^{1}, \ldots, \theta_{m}^{n}\right)$ are bargaining equilibrium proposals in the game with continuation probability $\delta_{m}$, such that $\left(\theta_{m}^{i}\right)_{m \in \mathbb{N}}$ converges to $\bar{\theta}$ for every $i \in N$.

Since the proposals $\theta_{m}^{i}$ in Lemma 6.1 all belong to the compact set $V_{+}$, every sequence $\left(\theta_{m}^{1}, \ldots, \theta_{m}^{n}\right)_{m \in \mathbb{N}}$ has a convergent subsequence. This demonstrates the existence of a limit bargaining equilibrium.

Corollary 6.3 A limit bargaining equilibrium exists. Each limit bargaining equilibrium is Pareto efficient.

Theorem 6.4 Let $\bar{\theta}$ be a limit bargaining equilibrium.

1. If $\pi_{i}^{i}=0$ for some $i \in N$, then $\bar{\theta}_{i}=0$.

2. Suppose there is exactly one player $i \in N$ with $\pi_{i}^{i}>0$. Then $\bar{\theta}_{i}$ is player $i$ 's utopia payoff $\hat{v}_{i}$ and $\bar{\theta}_{j}=0$ for $j \neq i$.

Proof: To prove Theorem 6.4.1, take a player $i \in N$ with $\pi_{i}^{i}=0$ and observe that in each bargaining equilibrium $\theta_{i}^{j}=0$ for every $j \neq i$. Taking the limit of $\theta_{m, i}^{j}$ along the appropriate sequence of bargaining equilibria yields $\bar{\theta}_{i}=0$. To prove Theorem 6.4 .2 , notice that $\bar{\theta}_{j}=0$ for $j \neq i$ by Theorem 6.4.1. The fact that $\bar{\theta}_{i}$ is player $i$ 's utopia payoff follows from the fact that $\bar{\theta}$ is a Pareto-efficient point of $V$.

We proceed by showing that the bargaining equilibrium is unique and is equal to the asymmetric Nash bargaining solution where player $i$ has weight $\pi_{i}^{i}$. Given a vector $\lambda \in$ $\mathbb{R}_{+}^{n} \backslash\{0\}$, we define the $\lambda$-Nash product $\rho_{\lambda}: \mathbb{R}_{+}^{n} \rightarrow \mathbb{R}$ by

$$
\rho_{\lambda}(v)=\prod_{i \in N} v_{i}^{\lambda_{i}}, \quad v \in \mathbb{R}^{n} .
$$

Definition 6.5 Given a vector $\lambda \in \mathbb{R}_{+}^{n} \backslash\{0\}$, the maximizer of the function $\rho_{\lambda}$ on $V_{+}$is called the $\lambda$-Nash bargaining solution. 
Under our assumptions the maximizer of the function $\rho_{\lambda}$ on $V_{+}$is indeed unique. It is a Pareto-efficient point of $V$ which is uniquely characterized by the following conditions:

$$
\begin{aligned}
& \lambda_{i}=0 \text { if and only if } v_{i}=0, \\
& \forall i, j \in N \text { such that } \lambda_{i}, \lambda_{j}>0, \frac{\eta_{i}(v)}{\eta_{j}(v)}=\frac{\lambda_{i} / v_{i}}{\lambda_{j} / v_{j}} .
\end{aligned}
$$

Theorem 6.6 The limit bargaining equilibrium is unique and is equal to the $\left(\pi_{1}^{1}, \ldots, \pi_{n}^{n}\right)$ Nash bargaining solution.

Proof: We verify that each limit bargaining equilibrium satisfies the conditions (6.1)-(6.2). Let

$$
\tilde{N}=\left\{i \in N \mid \pi_{i}^{i}>0\right\}
$$

Let $\left(\theta^{1}, \ldots, \theta^{n}\right)$ be a bargaining equilibrium in a game with continuation probability $\delta$. By the definition of the normal vector it holds for any two players $i$ and $j$ that

$$
\left(\theta^{j}-\theta^{i}\right)^{\top} \eta\left(\theta^{i}\right) \leq 0 .
$$

Notice that the proposals $\theta^{i}$ and $\theta^{j}$ can only differ in components $i$ and $j$. Solving for the inner product, we can therefore rewrite the previous inequality as

$$
\left(\theta_{j}^{j}-\theta_{j}^{i}\right) \eta_{j}\left(\theta^{i}\right)+\left(\theta_{i}^{j}-\theta_{i}^{i}\right) \eta_{i}\left(\theta^{i}\right) \leq 0 .
$$

Substituting for $\theta_{j}^{i}$ and $\theta_{j}^{j}$ from equation (4.2) and dividing by $1-\delta$ yields

$$
\frac{\theta_{j}^{j} \eta_{j}\left(\theta^{i}\right)}{1-\delta+\delta \pi_{j}^{j}} \leq \frac{\theta_{i}^{i} \eta_{i}\left(\theta^{i}\right)}{1-\delta+\delta \pi_{i}^{i}}
$$

Let $\bar{\theta}$ be a limit bargaining equilibrium. Then the previous inequality yields for all $i, j \in \tilde{N}$

$$
\frac{\bar{\theta}_{j} \eta_{j}(\bar{\theta})}{\pi_{j}^{j}} \leq \frac{\bar{\theta}_{i} \eta_{i}(\bar{\theta})}{\pi_{i}^{i}} .
$$

Interchanging the roles of the players $i$ and $j$, we obtain the equality

$$
\frac{\bar{\theta}_{j} \eta_{j}(\bar{\theta})}{\pi_{j}^{j}}=\frac{\bar{\theta}_{i} \eta_{i}(\bar{\theta})}{\pi_{i}^{i}}, \quad i, j \in \tilde{N} .
$$

By Corollary 6.4.1 we have that

$$
\bar{\theta}_{i}=0, \quad i \in N \backslash \tilde{N} .
$$

It only remains to be shown that $\bar{\theta}_{i}>0$ for every $i \in \tilde{N}$. Suppose to the contrary that $\bar{\theta}_{i}=0$ for some $i \in \tilde{N}$. Equation (6.3) and the fact that $\eta_{j}(\bar{\theta})>0$ for all $j \in \tilde{N}$ imply that $\bar{\theta}_{j}=0$ for all $j \in \tilde{N}$. Combining this with (6.4) we have $\bar{\theta}=0$, which contradicts the Pareto-efficiency of $\bar{\theta}$. 


\section{Example}

The analysis of bargaining equilibria reveals two rather striking features. First, only the "diagonal" probabilities $\pi_{1}^{1}, \ldots, \pi_{n}^{n}$ are relevant for the bargaining equilibrium payoffs, together with the initial probability to propose $\pi^{0}$ in case $\pi_{1}^{1}=\cdots=\pi_{n}^{n}=0$. The chance to become a proposer after a rejection by another player is not a source of bargaining power. A player whose probability to become a proposer after his own rejection is zero, will never be offered a positive payoff. Second, the bargaining equilibrium payoffs exhibit a discontinuity when all the probabilities $\pi_{1}^{1}, \ldots, \pi_{n}^{n}$ are equal to zero. In that case, whoever is the proposer in the initial round can appropriate the entire surplus, which is no longer the case when $\pi_{i}^{i}$ is non-zero for at least one $i \in N$.

The following example illustrates these two features. There are four players, $N=$ $\{1,2,3,4\}$. The vectors $\pi^{1}, \ldots, \pi^{4}$ are given by

$$
\left(\pi^{1}, \pi^{2}, \pi^{3}, \pi^{4}\right)=\left(\begin{array}{cccc}
0 & 1 & 1 & 1-\varepsilon \\
1 & 0 & 0 & 0 \\
0 & 0 & 0 & 0 \\
0 & 0 & 0 & \varepsilon
\end{array}\right)
$$

We first assume that $\varepsilon>0$ and suppose that the initial proposer is player 4 with probability zero. Player 4 appears very weak in the example at hand. In order for player 4 to become the proposer at all, some proposal must be accepted by players 1, 2, and 3 and then be rejected by player 4 . Even in that case, player 4 becomes the next proposer only with probability $\varepsilon$, which can be arbitrarily close to zero. Player 1 on the other hand, could be the initial proposer with probability as high as one, becomes the next proposer for sure whenever player 2 or player 3 makes a rejection, and becomes the next proposer with probability $1-\varepsilon$ whenever player 4 rejects. Nevertheless, our convergence result implies that player 4 has all the bargaining power in the limit bargaining equilibrium and receives his utopia payoff. On the contrary, players 1,2 , and 3 receive zero payoffs in the limit bargaining equilibrium.

Assume next that $\varepsilon=0$. In that case, we no longer have the convergence result presented in Section 6, but rather know from Section 5 that the initial distribution $\pi^{0}$ determines the equilibrium payoffs, which, moreover, are independent from the continuation probability $\delta$. Since we assumed that player 4 is the initial proposer with probability zero, he now receives a bargaining equilibrium payoff of zero. In the example at hand, player 3 appears to be a "weak" player in the sense that he can never become a proposer except in the very first round. One would expect that if $\delta$ is large enough, player 3's bargaining power vanishes. However, irrespective of the value of $\delta$, in equilibrium player 3 obtains his utopia payoff when he is selected as the initial proposer. 


\section{Conclusion}

We have considered multilateral bargaining games with endogenous protocols. The identity of the player who rejects the current proposal determines the probability distribution from which the next proposer is drawn. Surprisingly, the probability with which a player proposes after another player's rejection turns out to be irrelevant for the prediction of equilibrium proposals and payoffs. The probability with which a player proposes after his own rejection is crucial for the bargaining equilibrium prediction. Our main results highlight a discrepancy between the case where, conditional on his rejection, the probability to make a counter-offer is zero for every player, and the case where for some player this probability is positive.

If the probability to make a counter-offer is zero for all players, then the equilibrium payoffs are determined by the utopia payoffs and the recognition probabilities in the initial round. Although immediate agreement is reached, the resulting equilibrium utilities are inefficient. Indeed, they do not converge to an asymmetric Nash bargaining solution. If one views the recognition probabilities merely as parameters of the model, one may think of the inefficiency and non-convergence of equilibrium utilities as results which hold true only in one degenerate case. However, the case where the probability to make a counter-offer is zero for all players appears more significant when one takes the perspective of a planner who designs a bargaining protocol. Such a designer might find it reasonable to assign to any rejector a zero recognition probability in order to discourage rejections. The result shows that the protocol designed in such a way does not ensure efficiency, quite to the contrary, the resulting bargaining equilibrium utilities are Pareto-inefficient.

In the case where at least one player does have a strictly positive probability to make a counter-offer, we obtain the convergence to an asymmetric Nash bargaining solution, where the weights are assigned to the players in proportion to their probabilities to make a counter-offer. In particular, if all players have the same strictly positive probability to make a counter-offer, then the bargaining equilibrium utilities converge to the symmetric Nash bargaining solution, irrespective of how large that probability actually is.

One rather surprising outcome of our analysis is that the probability to become proposer conditional on the rejection of another player does not affect bargaining equilibrium payoffs at all. This also sheds new light on the findings in Miyakawa (2008) and Laruelle and Valenciano (2008) concerning the protocol with time-invariant recognition probabilities, which is a special case of our model. Under the time-invariant recognition probabilities, it is impossible to discern the effect of the probability to become proposer after one's own rejection as opposed to the probability to propose after another player's rejection. Our more general setup makes the importance of this distinction apparent. 


\section{APPENDIX. Flexible Response Orders}

Throughout the paper, we have assumed that players respond to a proposal in the fixed order $1, \ldots, n$. The purpose of the appendix is to explain that this assumption is without loss of generality. That is, the results remain true if one allows for the order of responses to be flexible. We make the following modifications to the model described in the main text. Let $\Sigma$ be the set of permutations on $N$. Suppose that $\pi^{0}, \pi^{1}, \ldots, \pi^{n}$ are probability distributions on the set $N \times \Sigma$. Assume that the proposer and the order of responses in round $t=0$ is drawn from $\pi^{0}$, and that the proposer and the order of responses in round $t>0$ is drawn from $\pi^{i}$ whenever player $i$ has rejected the proposal in round $t-1$. In this game, a stationary strategy for player $i$ consists of a proposal $\theta^{i, \sigma}$ for every $\sigma \in \Sigma$, and of an acceptance set $A^{j, \sigma, i}$ for every $(j, \sigma) \in N \times \Sigma$. We refer to the intersection $\cap_{i \in N} A^{j, \sigma, i}$ as the social acceptance set $A^{j, \sigma}$. Each profile of stationary strategies $(\Theta, \mathcal{A})$ induces continuation payoffs that are independent of the identity of the current proposer and of the current order of responses. Let $Q$ denote the $(n \times n)$-matrix of continuation payoffs and $r$ the $n$-vector of reservation payoffs, which corresponds to the diagonal of $Q$. We denote the $k^{\text {th }}$ element of the permutation $\sigma$ by $\sigma_{k}$, and we write $S(\sigma, k)=\left\{\sigma_{k}, \sigma_{k+1}, \ldots, \sigma_{n}\right\}$. The intersection $\cap_{j \in S(\sigma, k)} A^{i, \sigma, j}$ is denoted by $A^{i, \sigma, S(\sigma, k)}$.

Lemma A.1 Let $(\Theta, \mathcal{A})$ be an SSPE inducing reservation payoffs $r$. For all $(i, \sigma) \in N \times \Sigma$, the following hold:

1. If $v$ is such that $v_{\sigma_{n}}>r_{\sigma_{n}}$, then $v \in A^{i, \sigma, \sigma_{n}}$.

2. For every $k=1, \ldots, n-1$, if $v \in A^{i, \sigma, S(\sigma, k+1)}$ and $v_{\sigma_{k}}>r_{\sigma_{k}}$, then $v \in A^{i, \sigma, \sigma_{k}}$.

3. For every $k=1, \ldots, n$, if $v \in A^{i, \sigma, S(\sigma, k)}$, then $v_{j} \geq r_{j}$ for all $j \in S(\sigma, k)$.

The proof of the statements in the lemma above is analogous to the proofs of Lemmas 3.1 and 3.3. The above implies that any payoff allocation which is strictly preferred by all players to $r$ will be socially accepted irrespective of the choice of $(i, \sigma)$. Moreover, any payoff allocation which is strictly worse than the reservation payoff for at least one player will be rejected irrespective of the choice of $(i, \sigma)$.

Lemma A.2 Let $(\Theta, \mathcal{A})$ be an SSPE inducing reservation payoffs $r$. Then it holds that $r \in V_{+}$ and there is $v \in V$ such that $v \gg r$.

Proof: The proof that $r \in V_{+}$is analogous to the proof of Lemma 3.5. We show next that there is $v \in V$ such that $v \gg r$. Suppose by way of contradiction that $r \in P_{+}$. Then, it follows from Lemma A.1 that $\cup_{(i, \sigma) \in N \times \Sigma} A^{i, \sigma} \subset\{r\}$. For every $i \in N$, the expected payoff of rejecting a proposal is $r_{i}$. But if only $r$ can ever be socially acceptable, then $r_{i}$ must be a convex combination of 0 and $r_{i}$, where the former has a weight of at least $1-\delta$. Hence, it holds for every $i$ that $(1-\delta) 0+\delta r_{i} \geq r_{i}$. Since $\delta<1$, we have $r_{i}=0$ for every $i \in N$. But $r=0$ does not belong to $P_{+}$, the desired contradiction. 
Lemma A.3 Let $(\Theta, \mathcal{A})$ be an SSPE. For all $(i, \sigma) \in N \times \Sigma$, it holds that $\theta^{i, \sigma} \in A^{i, \sigma}$.

Proof: Suppose that for some $\left(i^{\prime}, \sigma^{\prime}\right) \in N \times \Sigma$, we have $\theta^{i^{\prime}, \sigma^{\prime}} \notin A^{i^{\prime}, \sigma^{\prime}}$. Let $j$ be the first player in the response order $\sigma^{\prime}$ to reject $\theta^{i^{\prime}, \sigma^{\prime}}$. Consider player $i^{\prime \prime}$ s continuation payoff $q_{i^{\prime}}^{j}$ after $j$ 's rejection of $i^{\prime}$ 's proposal. We argue first that $q_{i^{\prime}}^{j}>0$. Every player can guarantee a zero payoff by rejecting all proposals. Thus, it holds that $q_{i^{\prime}}^{j} \geq 0$. By Lemma A.2 it holds that there is $v \in V_{+}$such that $v \gg r$. By Lemma A.1 it holds that $v \in A^{i, \sigma}$ for every $(i, \sigma) \in N \times \Sigma$, so player $i^{\prime}$ can guarantee himself a strictly positive payoff by proposing such a $v$. Making the unacceptable proposal $\theta^{i^{\prime}, \sigma^{\prime}}$ must at least yield such a payoff, so $q_{i^{\prime}}^{j}>0$. This implies that for some $(i, \sigma) \in N \times \Sigma$, it must hold that $\theta^{i, \sigma} \in A^{i, \sigma}$. We can write $i^{\prime}$ 's expected payoff after $j$ 's rejection of $\theta^{i^{\prime}, \sigma^{\prime}}$ as $q_{i^{\prime}}^{j}=\hat{\delta} \hat{\theta}_{i^{\prime}}$, where $\hat{\delta} \leq \delta$ and $\hat{\theta}$ is a convex combination of proposals $\theta^{i, \sigma}$ for which it holds that $\theta^{i, \sigma} \in A^{i, \sigma}$. Given that $\hat{\delta} \leq \delta<1$, there must be some $\left(i^{\prime \prime}, \sigma^{\prime \prime}\right) \in N \times \Sigma$ such that $\theta^{i^{\prime \prime}, \sigma^{\prime \prime}} \in A^{i^{\prime \prime}, \sigma^{\prime \prime}}$ and $\theta_{i^{\prime}}^{i^{\prime \prime}}, \sigma^{\prime \prime}>q_{i^{\prime}}^{j}$. But the fact that $\theta^{i^{\prime \prime}, \sigma^{\prime \prime}}$ is accepted implies $\theta^{i^{\prime \prime}, \sigma^{\prime \prime}} \geq r$. So there is $\bar{v} \in V_{+}$such that $\bar{v}_{i^{\prime}}>q_{i^{\prime}}^{j}$ and $\bar{v}_{j}>r_{j}$ for all $j \in N \backslash\left\{i^{\prime}\right\}$. By Lemma A.1 it holds that $\bar{v} \in A^{i^{\prime}, \sigma^{\prime}, j}$ for all $j \in N \backslash\left\{i^{\prime}\right\}$. But then, player $i^{\prime}$ has a profitable deviation by proposing $\bar{v}$ instead of $\theta^{i^{\prime}, \sigma^{\prime}}$, a contradiction since $(\Theta, \mathcal{A})$ is an $\operatorname{SSPE}$.

Lemma A.4 Let $(\Theta, \mathcal{A})$ be an SSPE inducing reservation payoffs $r$. For every $(i, \sigma) \in N \times \Sigma$ it holds that $\theta^{i, \sigma} \in P_{+}$and $\theta_{j}^{i, \sigma}=r_{j}$ for all $j \in N \backslash\{i\}$.

The proof of this statement is analogous to the proof of Lemma 3.7 in the main text.

Since the sets of weakly and strongly Pareto-efficient points in $V_{+}$coincide, Lemma A.4 implies that the proposal $\theta^{i, \sigma}$ is independent of the response order $\sigma \in \Sigma$ and we denote the proposal by player $i$ by $\theta^{i}$. Acceptance sets, on the other hand, can depend on the responder order $\sigma$.

Now consider the reservation payoff $r_{i}$. We have shown the immediate agreement property in Lemma A.3. Thus, we can write

$$
r_{i}=\delta \sum_{(j, \sigma) \in N \times \Sigma} \pi_{j, \sigma}^{i} \theta_{i}^{j}
$$

We define $\pi_{j}^{i}=\sum_{\sigma \in \Sigma} \pi_{j, \sigma}^{i}$, and obtain that

$$
r_{i}=\delta \sum_{j \in N} \pi_{j}^{i} \theta_{i}^{j}=\delta \pi_{i}^{i} \theta_{i}^{i}+\delta\left(1-\pi_{i}^{i}\right) r_{i} .
$$

Rearranging this equality yields that $r_{i}=\alpha_{i} \theta_{i}^{i}$. Together with Lemma A.3 and A.4, we have obtained Conditions (3.4)-(3.6) of Theorem 3.9 for the more general protocol in the Appendix. Now it is straightforward to extend all the results in Sections 4, 5, and 6 to this more general protocol. 


\section{References}

Banks, J., And J. Duggan (2000), "A Bargaining Model of Collective Choice," American Political Science Review, 94, 73-88.

Binmore, K., A. Rubinstein, And A. Wolinsky (1986), "The Nash Bargaining Solution in Economic Modelling," Rand Journal of Economics, 17, 176-188.

Bloch, F. (1996), "Sequential Formation of Coalitions in Games with Externalities and Fixed Payoff Division," Games and Economic Behavior, 14, 90-123.

Bloch, F., And E. Diamantoudi (2011), "Non-cooperative Formation of Coalitions in Hedonic Games," International Journal of Game Theory, 40, 263-280.

Britz, V., P.J.J. Herings, And A. Predtetchinski (2010), "Non-cooperative Support for the Asymmetric Nash Bargaining Solution," Journal of Economic Theory, 145, 1951-1967.

Chate, S., And J.-A. Yang (1994), "An N-Person Pure Bargaining Game," Journal of Economic Theory, 62, 86-102.

Chatterjee, K., B. Dutta, D. Ray, and K. Sengupta (1993), "A Non-cooperative Theory of Coalitional Bargaining," Review of Economic Studies, 60, 463-477.

Duggan, J. (2011), "Coalitional Bargaining Equilibria," Working Paper, 1-33.

Haller, H. (1986), "Non-cooperative Bargaining of $N \geq 3$ Players," Economics Letters, 22, 11-13.

Hart, S., ANd A. Mas-Colell (1996), "Bargaining and Value," Econometrica, 64, 357-380.

Herings, P.J.J., And H. Houba (2010), "The Condorcet Paradox Revisited," METEOR Research Memorandum 10/09, Maastricht University, Maastricht, pp. 1-45.

Herings, P.J.J., And A. Predtetchinski (2011), "On the Asymptotic Uniqueness of Bargaining Equilibria," Economics Letters, 111, 243-246.

Herrero, M.J. (1985), A Strategic Bargaining Approach to Market Institutions, PhD Thesis, London School of Economics.

Imai, H., And H. Salonen (2000), "The Representative Nash Solution for Two-sided Bargaining Problems," Mathematical Social Sciences, 39, 349-365.

Kalai, E. (1977), "Nonsymmetric Nash Solutions and Replications of 2-Person Bargaining," International Journal of Game Theory, 6, 129-133.

Kalandrakis, T. (2006), "Proposal Rights and Political Power," American Journal of Political Science, $50,441-448$.

Kawamori, T. (2008), "A Note on Selection of Proposers in Coalitional Bargaining," International Journal of Game Theory, 37, 525-532.

Knight, B. (2005), "Estimating the Value of Proposal Power," American Economic Review, 95, 16391652.

Krishna, V., And R. Serrano (1996), "Multilateral Bargaining," Review of Economic Studies, 63, $61-80$. 
Kultti, K., And H. Vartiainen (2010), "Multilateral Non-Cooperative Bargaining in a General Utility Space," International Journal of Game Theory, 39, 677-689.

Miyakawa, T. (2008), "Noncooperative Foundation of $n$-Person Asymmetric Nash Bargaining Solution," Journal of Economics of Kwansei Gakuin University, 62, 1-18.

Laruelle, A., And F. Valenciano (2008), "Noncooperative Foundations of Bargaining Power in Committees and the Shapley-Shubik Index," Games and Economic Behavior, 63, 341-353.

Moldovanu, B., And E. Winter (1995), "Order Independent Equilibria," Games and Economic Behavior, 9, 21-34.

NASh, J.F. (1950), "The Bargaining Problem," Econometrica, 18, 155-162.

NASH, J.F. (1953), "Two-Person Cooperative Games," Econometrica, 21, 128-140.

OkAdA, A. (1996), "A Noncooperative Coalitional Bargaining Game with Random Proposers," Games and Economic Behavior, 16, 97-108.

Okada, A. (2011), "Coalitional Bargaining Games with Random Proposers: Theory and Application," Games and Economic Behavior, 73, 227-235.

Ray, D., And R. Vohra (1999), "A Theory of Endogenous Coalition Structures," Games and Economic Behavior, 26, 286-336.

Romer, T., And H. Rosenthal (1978), "Political Resource Allocation, Controlled Agendas, and the Status Quo," Public Choice, 33, 27-43.

Rubinstein, A. (1982), "Perfect Equilibrium in a Bargaining Model," Econometrica, 50, 97-109.

Selten, R. (1981), "A Noncooperative Model of Characteristic Function Bargaining," in V. Böhm and H.H. Nachtkamp (eds.), Essays in Game Theory and Mathematical Economics in Honor of Oskar Morgenstern, Bibliografisches Institut Mannheim, pp. 131-151.

Suh, S.-C., And Q. Wen (2006), "Multi-agent Bilateral Bargaining and the Nash Bargaining Solution," Journal of Mathematical Economics, 42, 61-73. 